Artículo Original

\title{
Nuevo género y especie de Anobiinae (Coleoptera: Ptinidae) de Chile central
}

New genus and species of Anobiinae (Coleoptera: Ptinidae) from central Chile

\author{
Alfredo Lüer ${ }^{1^{*}}$ y Richard Honour ${ }^{2}$
}

${ }^{1}$ Panguilemo 261, Quilicura, Santiago, Chile. ${ }^{*}$ alfredoluer@hotmail.com. ${ }^{2}$ Investigador externo área de Entomología, Museo Nacional de Historia Natural, Santiago, Chile. E-mail: rhonour@gmail.com

\section{ZooBank: urn:lsid:zoobank.org:pub:AB80565C-4953-40AA-B88E-BEB2DB69B625 https: / / doi.org/10.35249/ rche.47.3.21.13}

Resumen. Se describe un nuevo género y especie de Anobiinae (Coleoptera: Ptinidae), Austronobium flavus gen. n. y sp. n., a partir de ejemplares recolectados y obtenidos de plantas hospedantes en Chile central. Se entregan caracteres diagnósticos de los taxones, incluido imágenes del adulto y de las estructuras genitales de ambos sexos.

Palabras clave: Nuevo taxón; Región Andina; taxonomía.

Abstract. A new genus and species of Anobiinae (Coleoptera: Ptinidae) are described, Austronobium flavus gen. n. and sp. n., based on specimens collected and obtained from host plants in central Chile. Diagnostic characters and images from adults and genitalia of both sexes are given.

Key words: Andean Region; new taxa; taxonomy.

\section{Introducción}

La subfamilia Anobiinae Fleming, 1821 (Coleoptera: Ptinidae) está compuesta a nivel mundial por 46 géneros, distribuidos en la mayoría de las regiones del mundo (Zahradník y Háva 2014). En Chile esta subfamilia está representada por 14 especies distribuidas en seis géneros (entre paréntesis número de especies en cada uno): Allobregmus Español, 1970 (3), Anobium Fabricius, 1775 (3), Hemicoelinum Español, 1971 (1), Leptanobium Español y Comas, 1988 (4), Megabregmus Español, 1970 (2) y Stegobium Motschulsky, 1860 (1), con registros en territorio continental e insular (Blackwelder 1945; Español 1971; Español y Blas 1991; González 1989; Honour y Rothmann 2018; Lüer 2020).

El objetivo de este trabajo es describir un nuevo género y especie de Anobiinae de Chile central.

\section{Materiales y Métodos}

Se examinaron 25 ejemplares recolectados mediante sacudido de follaje, uso de trampa de luz y crianza de estados inmaduros (a partir de madera muerta infestada). El aparato genital fue extraído y limpiado en una solución de hidróxido de potasio al 20\% por 15 minutos, y para la diafanización de las estructuras muy esclerotizadas se utilizó ácido

Recibido 26 Julio 2021 / Aceptado 19 Agosto 2021 / Publicado online 31 Agosto 2021 Editor Responsable: José Mondaca E. 
acético al 99\% durante dos a cuatro minutos. El largo corporal fue medido en vista dorsal, desde el borde anterior del pronoto hasta el ápice de los élitros, y el ancho máximo fue medido en la parte más amplia de los élitros. El mapa de distribución se generó a partir del sitio web SimpleMappr (http://www.simplemappr.net/). Las fotografías del adulto fueron tomadas con el espécimen fijado en una plataforma Nikon PB-6M por medio de un lente Nikon M Plan 50.1 210 / 0 acoplado a un fuelle Nikon PB-6 y este a una cámara Nikon D7000, y para las imágenes de microscopio se utilizó una cámara de video MiniVid que reemplaza al ocular del microscopio.

El material tipo queda depositado en las siguientes colecciones entomológicas: Museo Nacional de Historia Natural, Santiago, Chile (MNNC); Colección Alfredo Lüer Hernández, Santiago, Chile (CALH); Colección Richard Honour Sepúlveda, Santiago, Chile (CRHS).

\section{Resultados}

\section{Austronobium gen. $\mathbf{n}$.}

(Figs. 1-12)

Especie tipo. Austronobium flavus sp. n., presente designación.

Diagnosis. Cuerpo más de dos veces largo que ancho, costados subparalelos; superficie con pilosidad densa que dificulta ver el tegumento. Puntuación general del cuerpo débil. Cabeza no visible en vista dorsal (Fig. 1), no excavada. Antena de once antenómeros, con maza de tres que supera la mitad del largo total (Figs. 2, 3). Pronoto más ancho que largo; disco con elevación moderada. Mesoventrito y metaventrito con proceso no excavado (Fig. 7). Élitros subparalelos, apicalmente redondeados y con serie de estrías longitudinales notorias (Fig. 1). Cavidades coxales separadas. Abdomen con primer ventrito más largo que el tercero; el segundo el más largo; sutura entre el ventrito I y II débil en el medio (Figs. 9, 10). Edeago simétrico; lóbulo medio de costados subparalelos, angostándose leve y progresivamente hacia el ápice; piezas accesorias de los parámeros largas (Fig. 11).

Descripción. Cuerpo más de dos veces máslargoqueancho, subparalelo, dorsoventralmente convexo; pilosidad corta, densa y apegada al tegumento (Fig. 1); tegumento levemente rugoso y con puntuación general débil. Cabeza: no visible en vista dorsal, no excavada ni deprimida por debajo. Ojos grandes, levemente sobresalientes. Antena de once antenómeros, con maza terminal de tres que supera la mitad del largo total de la antena (Figs. 2, 3). Último segmento del palpo maxilar alargado, con borde exterior recto e interior en suave curva convexa (Fig. 4). Último segmento del palpo labial subtriangular (Fig. 5). Tórax: pronoto en vista dorsal moderadamente más ancho que largo, con su mayor ancho en la base y menor ancho en el centro; ángulos posteriores agudos (Fig. 1a), reborde lateral completo, basalmente expandido; disco con elevación moderada. Prosterno con proceso medial laminar ancho y margen apical ampliamente hendido en el centro, conformando dos proyecciones aguzadas (Fig. 6). Mesoventrito medialmente con área anterior oval, cóncava, no excavada; entre coxas con área levemente deprimida y de bordes realzados, no excavada. Metaventrito con proceso intercoxal tan largo como ancho, no excavado, de borde rectangular y realzado (Fig. 7). Cavidades coxales separadas. Patas largas; trocánter un poco más largo que ancho, fémur fusiforme, con lado interno excavado para recibir parte de la tibia; tibia con espolones internos; tarsos con los dos primeros segmentos alargados, el primero el más largo; garras pretarsales simples (Fig. 8). Escutelo transverso y rectangular. Élitros subparalelos, basalmente de ancho similar al ancho máximo del pronoto, apicalmente estrechándose y con su extremo redondeado; con serie de estrías marcadas, algunas de estas reunidas apicalmente antes del declive. Abdomen: los dos 
primeros ventritos más largos, siendo el segundo el mayor; sutura entre el ventrito I y II débil en el medio (Figs. 9, 10). Edeago simétrico, largo, con lóbulo medio subparalelo y pieza accesoria ciliada (Fig. 11). Ovipositor alargado, con coxitos y estilos desarrollados (Fig. 12).

Etimología. Del latín austro (sur), en referencia a la ubicación biogeográfica del nuevo taxón, y Anobium, por la similitud morfológica que presentan ambos géneros.

Comentario. Austronobium gen. n. se asemeja a los géneros agrupados por Español y Blas (1991) en la sección Anobium, compuesta por Anobium, Cacotemnus LeConte, 1861, Hemicoelinum, Hemicoelus LeConte, 1861 y Platybregmus Fisher, 1934, por la forma general del cuerpo, tener antenas de once antenómeros con maza terminal de tres, presentar primer ventrito abdominal tan o más largo que el tercero y por el edeago simétrico. Se separa de estos géneros, principalmente, por la diferente conformación del pronoto, por tener estrías elitrales con puntuación muy débil, presentar sutura entre el ventrito I y II débil en el medio, y por el tipo de edeago, que presenta parámeros subparalelos, alargados y sin expansiones.

Respecto a otros géneros de Anobiinae presentes en Chile, Austronobium gen. n. se diferencia de Allobregmus y Megabregmus por presentar metaventrito anteriormente no excavado y edeago simétrico, entre otros caracteres; de Leptanobium se separa por presentar maza antenal y estrías elitrales marcadas, y de Stegobium por poseer cavidades mesocoxales separadas y edeago simétrico.

\section{Austronobium flavus sp. $\mathbf{n}$.}

(Figs. 1-12)

Diagnosis. Cuerpo ente 2,4-2,6 veces más largo que ancho. Color general marrón rojizo oscuro; pilosidad densa, corta y apegada al tegumento, de tonalidad amarillenta con reflejo dorado. Maza antenal ocupa un poco más del 60\% del largo total de la antena en machos (Fig. 2) y ligeramente menos del $60 \%$ en hembras (Fig. 3). Pronoto con borde anterior elevado detrás de los ojos. Proceso mesoventral con área cordiforme (Fig. 7). Edeago con lóbulo medio ancho en la base, angostándose hacia el tercio apical y ligeramente redondeado en el ápice; saco interno medialmente con piezas esclerosadas alargadas, dos subparalelas y dos contiguas más basales (Fig. 11).

Descripción holotipo macho. Longitud: 2,51 mm; ancho: 0,98 mm. Cuerpo marrón rojizo oscuro, algo más claro en apéndices y ventritos; pilosidad amarillenta con reflejo dorado, densa, corta y apegada al tegumento (Fig. 1). Cabeza: frente regularmente convexa, superficie rugoso-punteada muy débil. Ojos grandes, ligeramente alargados y suavemente escotados centralmente por la base de la antena, separados 1,8 veces el diámetro vertical de un ojo. Mandíbula de igual ancho que largo, con dos dientes evidentes y un tercero insinuado; diente apical aguzado. Antenas estiradas hacia atrás alcanzan los 2/ 3 del metaventrito; escapo largo, algo curvado y 1,4 veces más largo que el antenómero II; antenómeros III al VIII cortos y transversos, V y VII ligeramente aserrados; maza antenal ocupa el 63,7\% del largo total de la antena; antenómero XI 1,2 veces más largo que segmento X y 1,5 veces más largo que el IX (Fig. 2). Último segmento del palpo maxilar con borde externo recto e interno en suave curva, 2,4 veces más largo que ancho y ancho máximo en los $2 / 3$ de la pieza (Fig. 4). Último segmento el palpo labial triangular, 0,2 veces más largo que ancho y borde apical ligeramente escotado (Fig. 5). Tórax: pronoto 1,2 veces más ancho que largo, mayor ancho en la base; margen anterior moderadamente elevado detrás de ojos; disco con granulación circular muy débil, separada de uno a dos 
veces el diámetro de un gránulo; reborde lateral sinuoso, basalmente expandido. Escutelo 1,6 veces más ancho que largo. Élitros en conjunto 1,9 veces más largos que anchos y 2,6 veces más largos que el pronoto, basalmente apenas más angostos que la base del pronoto; lados paralelos en los $3 / 4$ basales. Superficie finamente rugosa; once estrías, diez subparalelas, que nacen casi en la base, y una escutelar corta y oblicua; estrías con puntuación rectangular muy débil, que se hacen algo más evidentes en el primer tercio elitral; interestrías algo convexas hacia los costados y ápice. Lámina prosternal 1,2 veces el ancho de una cavidad procoxal (Fig. 6). Mesoventrito con área anterior medial cóncava, de superficie lisa y de suave margen realzado. Ancho del proceso mesoventral cerca de 0,5 veces el ancho de la respectiva cavidad coxal; área cordiforme entre coxas con márgenes fuertemente realzados. Metaventrito con proceso de ancho similar al proceso mesoventral; margen posterior con hendidura longitudinal media angosta y profunda; superficie rugoso-punteada, más notoria hacia los costados; puntos débiles, redondos, separados de 0,5 a tres veces el diámetro de un punto (Fig. 7). Patas con dos espolones tibiales pequeños; primer tarsómero 1,6 veces más largo que el tarsómetro II, y este último, 0,6 veces el largo de los tres siguientes en conjunto (Fig. 8). Abdomen: 1,2 veces más largo que ancho, mayor ancho en ventrito II. Ventrito I (excluido el proceso intercoxal) y II los más largos, el primero 0,9 veces el largo del segundo; ventrito III 1,3 veces más largo que el IV, este el más corto y 0,8 veces el largo del V; primera sutura ligeramente arqueada hacia atrás (Fig. 9); superficie del primer ventrito con granulación similar a la del metaventrito; II al V con puntuación muy fina, separada de una a cuatro veces el diámetro de un punto. Edeago: longitud 0,62 mm; ancho máximo 0,26 mm. Parámeros rectos con pieza accesoria que se ensancha progresivamente hacia el ápice. Lóbulo medio ancho en la base, angostándose hacia el tercio apical, con ápice suavemente redondeado, medialmente con piezas esclerosadas alargadas; dos subparalelas y hacia la base dos contiguas (Fig. 11).

Hembra. Similar al macho. Distancia interocular 2,0 veces el diámetro vertical de un ojo. Antenas de largo relativo similar al de los machos; escapo oblongo, levemente curvado y 1,8 veces más largo que el antenómero II; antenómeros III al VIII transversos y ligeramente aserrados, con expansión más pronunciada en antenómeros V y VII; maza antenal equivalente al 58\% del largo total de la antena; antenómero XI 4,1 veces más largo que ancho y 1,4 veces más largo que los segmentos X y IX (Fig. 3). Abdomen 1,2 veces más largo que ancho, mayor ancho en ventrito II. Ventrito I y II los más largos, el primero 0,7 veces el largo del segundo; ventrito III 1,3 veces más largo que el IV, este el más corto, y 0,9 veces el largo del V; primera sutura recta, no arqueada (Fig. 10). Ovipositor de 0,8 mm de largo, 3,2 veces más largo que ancho; gonocoxitos de un 31\% el largo total del ovipositor (Fig. 12).

Variabilidad. Machos ( $\mathrm{n}=10)$ : largo: 2,2-2,6 mm; ancho: 0,9-1,0 mm. Hembras ( $\mathrm{n}=10)$ : largo: 2,8-4,1 mm; ancho: 1,1-1,5 mm. La coloración en algunos ejemplares es de tonalidad más clara, especialmente en apéndices y ventritos.

Etimología. Del latín flavus (amarillo, dorado), en referencia a la pubescencia que presenta la especie.

Material tipo. Holotipo: 1 macho. Chile, Provincia de Cordillera, Pirque, emerge 17XI-2019, leg. A. Lüer. / Madera muerta de Hedera canariensis, recogida el 1-III-2018. Depositado en MNNC. Paratipos: 2 ejemplares. Chile, Provincia de Choapa, Los Vilos, emerge 15-XII-2016, leg. A. Lüer. / Madera muerta de Fuchsia lycioides, recogida el 30X-2016; 2 ejemplares. Chile, Provincia de Choapa, Los Vilos, emerge 2-XII-2016, leg. A. Lüer. / Madera muerta de Lobelia polyphylla, recogida el 30-X-2016; 3 ejemplares. Chile, 
Provincia de Valparaíso, Mantagua, emerge 25-XI-2015, leg. A. Lüer. / Madera muerta de Lobelia excelsa, recogida el 21-XI-2015; 4 ejemplares. Chile. R. de Valparaíso, Olmué, 4-I2014, leg. J.F. Campodonico. / Lámpara UV; 3 ejemplares. Chile, Provincia de Quillota, Hijuelas, emerge 25-XI-2018, leg. A. Lüer. / Madera muerta de Cestrum palqui, recogida el 17-IX-2018; 3 ejemplares. Chile. Provincia de Talagante, Cerro Naltagua, emerge 20-XII2016, leg. A. Lüer. / Madera muerta de Lobelia excelsa, recogida el 14-II-2016; 1 ejemplar. Chile. Provincia de Talagante, Naltagua, 3-XI-2013, leg. A. Lüer. / Follaje Lobelia excelsa; 2 ejemplares. La Ligua, Quebrada del Pobre, 02-I-2010. Leg. R. Honour; 1 ejemplar. San José de Maipo, Las Vertientes, II-2017, luz negra. Leg.: P. Bosch; 2 ejemplares. Chile, Provincia de Cordillera, Pirque, emerge 17-XI-2019, leg. A. Lüer. / Madera muerta de Hedera canariensis, recogida el 1-XII-2018; 1 ejemplar. Chile, Provincia de Curicó, Los Queñes, emerge 10-XI-2016, leg. A. Lüer. / Madera muerta de Escallonia pulverulenta, recogida el 9-X-2016; 1 ejemplar. Chile, Provincia de Cauquenes, Pelluhue, emerge 13-X-2018, leg. A. Lüer. / Madera muerta de Retama sphaerocarpa, recogida el 29-X-2017. Depositados en MNNC (5 ej.); CALH (10 ej.); CRHS (10 ej.).

Material adicional estudiado. 5 ejemplares. Chile, Choapa, Los Vilos, emerge 2-XII-2016, leg. A. Lüer. / Madera muerta de Fuchsia lycioides, recogida el 31-X-2016; 10 ejemplares. Chile, Provincia de Valparaíso, Mantagua, emerge 25-XI-2015, leg. A. Lüer. / Madera muerta de Lobelia excelsa, recogida el 21-XI-2015; 5 ejemplares. Chile, Provincia de Quillota, Hijuelas, emerge 25-XI-2018, leg. A. Lüer. / Madera muerta de Cestrum palqui, recogida el 17-IX-2018; 8 ejemplares. Chile. Provincia de Talagante, Cerro Naltagua, emerge 20-XII-2016, leg. A. Lüer. / Madera muerta de Lobelia polyphylla, recogida el 14-II2016; 3 ejemplares. Chile, Provincia de Cordillera, Pirque, emerge 1-XII-2018, leg. A. Lüer. / Madera muerta de Hedera canariensis, recogida el 1-XII-2018. Depositados en MNNC (10 ej.); CALH (11 ej.); CRHS (10 ej.).

Distribución y aspectos biológicos. A. flavus sp. n., se conoce de la Región de Coquimbo (Los Vilos), de Valparaíso (Mantagua, Olmué, Quebrada del Pobre, Hijuelas), Metropolitana de Santiago (Cerro Naltagua, Las Vertientes, Pirque) y del Maule (Los Queñes, Pelluhue) (Figs. 13, 14). Estas localidades se insertan, de acuerdo con Morrone (2015), en las provincias biogeográficas de Coquimbo y Santiago, de la Subregión Chilena Central, Región Andina.

La mayoría de los ejemplares fueron obtenidos por crianza de madera muerta, en tallos de Cestrum palqui L'Herit. (Solanaceae) (Fig. 15), Escallonia pulverulenta (Ruiz et Pav.) (Escalloniaceae), Fuchsia lycioides Andrews (Onagraceae), Hedera canariensis (Willd.) DC. (Araliaceae), Lobelia excelsa Bonpl., L. polyphylla Hook. y Arn. (Campanulaceae) y Retama sphaerocarpa L. (Fabaceae). Ocasionalmente se recolectaron adultos por sacudido de follaje de Lobelia excelsa o sin identificación de planta. Cinco ejemplares fueron capturados con luz artificial (luz negra, UV), lo que sugiere hábitos nocturnos de la especie.

La distribución temporal de adultos es la siguiente: por sacudido de follaje y trampa de luz: enero (6), febrero (1); por crianza: octubre (1), noviembre (26), diciembre (23).

Comentario. En Chile, Austronobium flavus sp. n. se separa de la especie alóctona Anobium punctatum Fabricius, 1775 por presentar esta especie, entre otros caracteres, puntuación elitral fuerte, alineada, formando estrías, tener meso y metaventrito excavados y por el distinto diseño del edeago. Respecto a las especies autóctonas mantenidas hasta ahora en Anobium, se diferencia de A. sulcatum Solier, 1849 por presentar esta especie cuerpo más corto (2,2 veces más largo que ancho), granulación y puntuación bien marcada del tegumento. De A. haemorrhoidale Philippi, 1864 se diferencia por el distinto diseño general del cuerpo, el mayor largo relativo de las antenas y la presencia de manchas rojizas elitrales en esta última especie. 

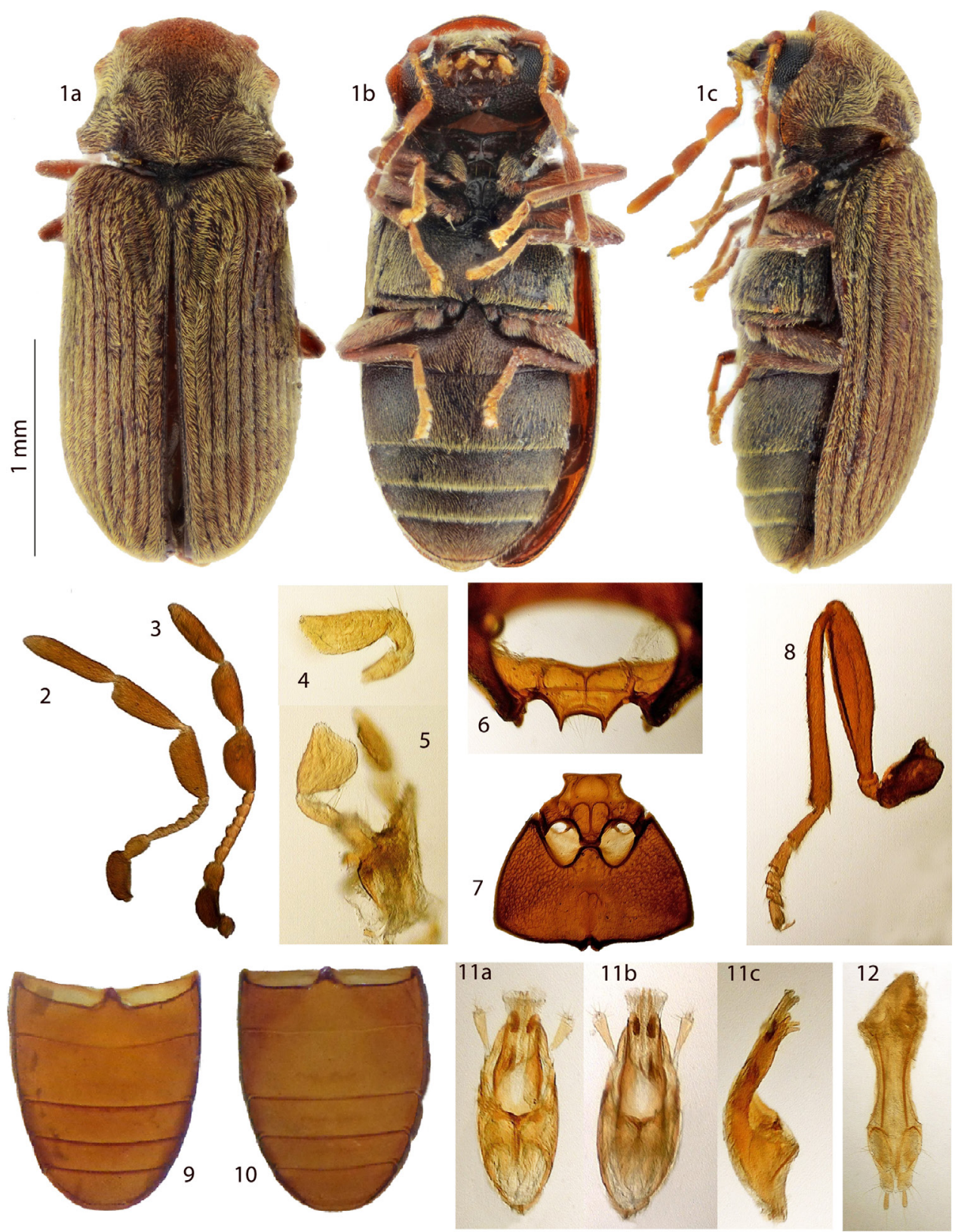

Figuras 1-12. Austronobium flavus sp. n. 1. Hábito en vista dorsal (a), ventral (b) y lateral (c). 2. Antena del macho. 3. Antena de la hembra. 4. Palpo maxilar. 5. Palpo labial. 6. Prosterno. 7. Mesoventrito y metaventrito. 8. Pata mesotorácica. 9. Abdomen del macho. 10. Abdomen de la hembra. 11. Edeago en vista dorsal (a), ventral (b) y lateral (c). 12. Ovipositor, vista ventral. / Austronobium flavus n. sp. 1. Habitus in dorsal (a), ventral (b) and lateral (c) view. 2. Antenna of the male. 3. Antenna of the female. 4. Maxillary palpus. 5. Labial palpus. 6. Prosternum. 7. Mesoventrite and metaventrite. 8. Mesothoracic leg. 9. Male abdomen. 10. Female abdomen. 11. Aedeagus in dorsal (a), ventral (b) and lateral (c) view. 12. Ovipositor in ventral view. 


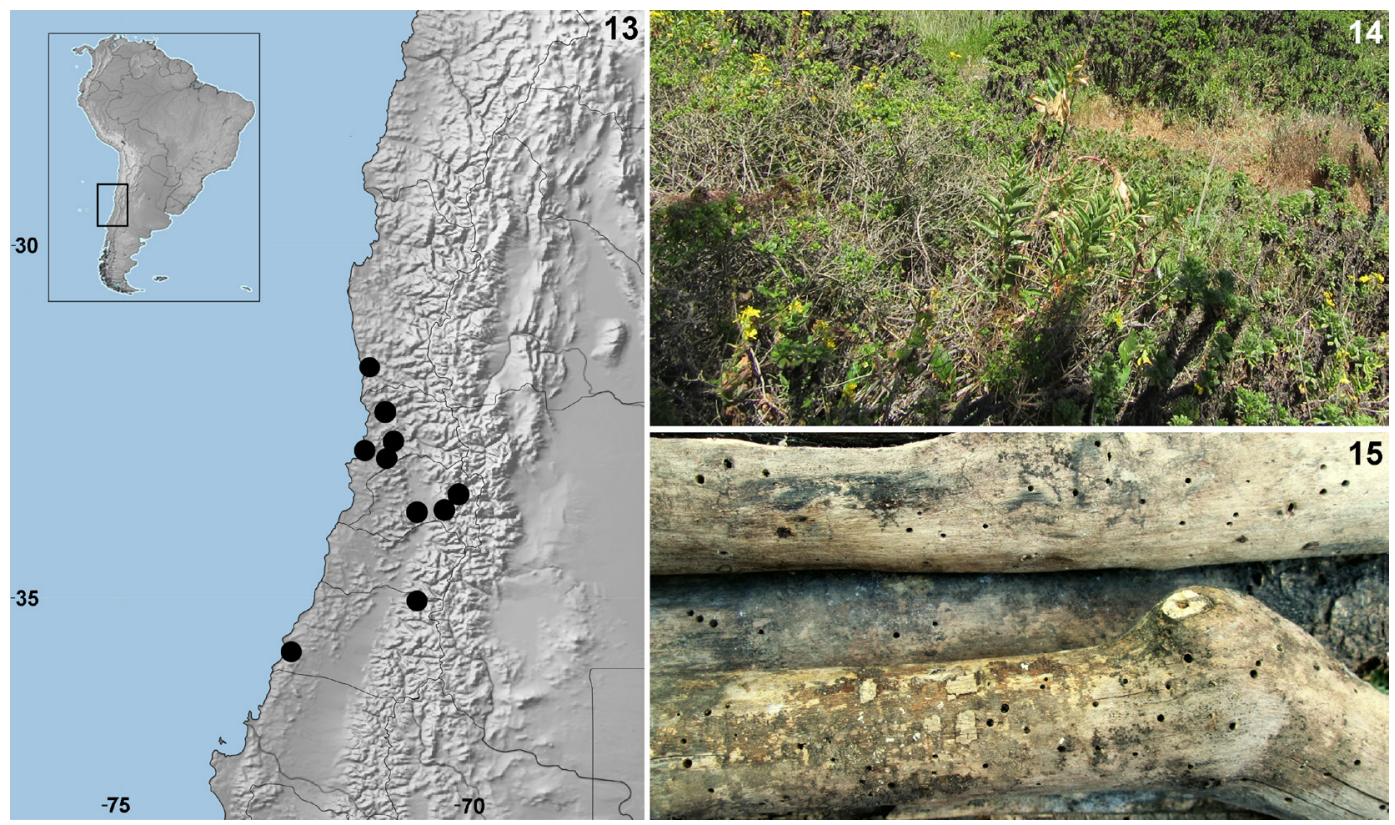

Figuras 13-15. Distribución geográfica y hábitat de Austronobium flavus sp. n. 13. Registros distribucionales. 14. Hábitat, Los Vilos, Provincia de Choapa, Región de Coquimbo. 15. Planta hospedante, madera muerta de Cestrum palqui con orificios de emergencia. / Geographical distribution and habitat of Austronobium flavus n. sp. 13. Distributional records. 14. Habitat, Los Vilos, Provincia de Choapa, Región de Coquimbo. 15. Host plant, deadwood of Cestrum palqui with emergence holes.

\section{Discusión}

Entre los géneros de Anobiinae presentes en Chile, A. flavus sp. n. posee similitud morfológica con Hemicoelinum, de Chile central, sur y austral, y también, aunque en menor grado, con Allobregmus, endémico de Chile, y Megabregmus, con representantes en Australia, Argentina, Chile y Brasil. A. flavus sp. n. presenta rasgos externos similares a las especies alóctonas Stegobium paniceum (Linnaeus, 1758) y Anobium punctatum DeGeer, 1774, compartiendo con esta última una mayor similitud morfológica.

El hecho de que $A$. flavus sp. n. haya pasado inadvertido hasta hoy podría ser explicado por el tamaño pequeño de la especie, hábitos nocturnos y distribución temporal de adultos relativamente restringida, aunque no se descarta que algún ejemplar fuese confundido con A. punctatum. Por otra parte, tomando en consideración que el grueso de los ejemplares estudiados se obtuvo por crianza de madera muerta, provenientes de seis localidades, resalta este método de recolecta como mecanismo necesario para la obtención de algunos Ptinidae poco conocidos, a la vez que permite inferir sobre la abundancia y distribución geográfica de las especies.

Con la incorporación de A. flavus sp. n., la subfamilia Anobiinae aumenta en el país a 15 especies en siete géneros, en los que resalta un alto grado de endemismo, con un 80,0\% a nivel de especie y $57,1 \%$ a nivel de género.

\section{Agradecimientos}

A Pablo Bercedo, por proporcionar parte de la bibliografía, y a Cristian Pineda, por realizar las fotografías del adulto de la especie. A Juan Francisco Campodonico, por la revisión crítica del manuscrito y ceder ejemplares de esta especie. 


\section{Literatura Citada}

Blackwelder, R. (1945) Checklist of the Coleopterous Insects of Mexico, Central America, the West Indies, and South America, Part. 3, U.S. National Museum Bulletin, 185: 343-550.

Español, F. (1971) Notas sobre Anóbidos (Col.) LIV. Sobre el complejo Anobium y otros elementos afines. Publicaciones del Instituto de Biología Aplicada, 50: 17-40.

Español, F. y Blas, M. (1991) Propuesta de una nueva ordenación genérica de los Anobiinae: Las secciones Anobium Fabricius y Priobium Motschulsky (Coleoptera: Bostrychoidea). Elytron Supplement, 5(1): 125-139.

González, R.H. (1989) Insectos y Ácaros de importancia Agrícola y Cuarentenaria en Chile. Ediciones Ograma Sociedad Anónima, 260 pp.

Honour, R. y Rothmann, S. (2018) Redefinición del género Leptanobium Español y Comas (Coleoptera: Ptinidae), con descripción de tres especies nuevas del norte de Chile. Revista Chilena de Entomología, 44(4): 475-485.

Lüer, A. (2020) Lista de plantas hospedantes de Ptinidae (Coleoptera: Bostrichoidea) de Chile. Revista Chilena de Entomología, 46(2): 333-344.

Morrone, J. (2015) Biogeographical regionalisation of the Andean region. Zootaxa, 3936(2): 207-236.

Zahradník, P. y Háva, J. (2014) Catalogue of the world genera and subgenera of the superfamilies Derodontoidea and Bostrichoidea (Coleoptera: Derodontiformia, Bostrichiformia). Zootaxa, 3754(4): 301-352. 